\title{
INTERAÇÃO DO FTALATO DE BENZILBUTIL COM BETA-CICLODEXTRINA E GRAFENO: UM ESTUDO DE PRIMEIROS PRINCÍPIOS
}

\author{
Jaine Schemmer ${ }^{1}$; Mariana Zancan Tonel2; Ivana Zanella ${ }^{3}$; Solange Binotto \\ Fagan $^{4}$
}

\section{RESUMO}

O objetivo deste trabalho é avaliar as propriedades estruturais, energéticas e eletrônicas da interação da beta-ciclodextrina ( $\beta-C D)$ e/ou grafeno com a molécula de ftalato de bezilbutil (BBP), através de simulações computacionais de primeiros princípios. Essas nanoestruturas podem ser usadas como possíveis nanofiltros para adsorção de ftalatos, pois a $\beta-C D$ é útil na formação de inclusão de complexos e o grafeno é um nanomaterial com uma grande área superficial que possibilita a adsorção de moléculas. A avaliação é baseada na Teoria do Funcional da Densidade (DFT) através do programa Siesta. Os resultados obtidos, mostram que os sistemas mais estáveis apresentaram valores de energia de ligação entre o BBP e o grafeno/ $\beta-C D$ de $0,42 \mathrm{eV} / 0,94 \mathrm{eV}$ respectivamente, ou seja, uma interação fraca. Dessa forma, esses sistemas são promissores para a remoção de moléculas de ftalatos.

Palavras-chave: Aplicações Ambientais, Nanomaterial, Simulação, DFT, Adsorção Física.

Eixo Temático: Tecnologia, Inovação e Desenvolvimento Sustentável (TIDS).

'Aluna de Iniciação Científica (PROBIC-Fapergs) do curso de Física Médica - Universidade Franciscana. E-mail: jaineschemmer33@gmail.com

${ }^{2}$ Colaboradora. Bolsista de pós-doutorado na Universidade Franciscana; marianaztonel@gmail.com

${ }^{3}$ Colaboradora. Docente do programa de Pós-graduação em Nanociências; ivanazanella@gmail.com

${ }^{4}$ Orientadora. Docente do programa de Pós-graduação em Nanociências. solange.fagan@gmail.com 


\section{INTRODUÇÃO}

Os ftalatos são ésteres orgânicos, amplamente utilizados na fabricação de plásticos, pois aumentam muito a plasticidade, resistência e flexibilidade dos produtos, reduzindo sua fragilidade. Também são utilizadas como aditivos na fabricação de borracha, têxteis, cosméticos e materiais de construção. No entanto, não existe uma ligação química entre as moléculas de ftalatos e as moléculas de polímero em produtos plásticos (OKAMOTO; UEDA; KOJIMA, 2011; ZHENG; ZHANG; TENG, 2014), os ftalatos são fáceis de dissolver, migrar e volatilizar produtos plásticos. As moléculas de ftalatos são onipresentes na atmosfera, água, sedimentos, solo e até mesmo organismos e em humanos (CHEN et al., 2012; KAEWLAOYOONG et al., 2018; ZHENG; ZHANG; TENG, 2014). A toxicidade das moléculas de ftalatos é atribuída principalmente à sua atividade semelhante ao estrogênio, que danifica o sistema endócrino de organismos e humanos e causa a disfunção reprodutiva do organismo (HU; YANG; WANG, 2015; ZHOU; CAI; XING, 2011). Por exemplo, o ftalato de benzilbutil (BBP), é uma substância química industrial amplamente utilizada, e está associada ao aumento do risco de câncer de próstata (DUAN et al., 2020). Devido ao grande uso industrial e ao risco ao meio ambiente, principalmente pelos seus efeitos adversos à saúde humana e animal, é fundamental a remoção adequada dessas moléculas, principalmente de meios aquáticos (BENJAMIN et al., 2017; GONZÁLEZ-SÁLAMO; SOCAS-RODRÍGUEZ; HERNÁNDEZ-BORGES, 2018; RADKE et al., 2019).

Já os nanomateriais têm atraído a atenção da comunidade científica devido às suas propriedades físico-químicas, mecânicas e elétricas diferenciadas. Tais propriedades tornam estes materiais nanoestruturados potencialmente promissores para aplicação em diversos campos da ciência, como no tratamento de efluentes devido à sua potencial capacidade de remoção de poluentes, como os ftalatos. Entre os nanomateriais existentes, dois foram escolhidos para serem utilizados na formação de sistemas com os ftalatos citados acima, sendo eles: ciclodextrina e 
grafeno (TERRONES et al, 2010).

As ciclodextrinas (CD), por sua vez, são oligossacarídeos cíclicos que possuem inúmeras aplicações (DEL VALLE, 2004; HEDGES, 1998). A beta-ciclodextrina $(\beta-C D)$ consiste em sete unidades de glucopiranose, sendo uma das mais estáveis (FIGUEIREDO et al., 2016). Sua estrutura molecular, que se aproxima de um cone truncado, gera uma superfície externa hidrofílica e interior da cavidade não polar. Dessa forma, as ciclodextrinas podem interagir adequadamente com moléculas, resultando na formação de inclusão de complexos. Esses complexos não covalentes podem oferecer uma variedade de vantagens físicas e químicas sobre algumas substâncias de interesse químico ou biológico, incluindo a possibilidade de aumento de solubilidade em água e estabilidade química.

Já o grafeno $(\mathrm{Gr})$ é formado por poucas camadas hexagonais, essas camadas que formam o grafite (NOVOSELOV, 2004). As propriedades eletrônicas e a grande área superficial são algumas das características mais interessantes para a adsorção de moléculas de interesse químico ou biológico (DE MORAES et al., 2019; VENDRAME et al., 2019; ZANELLA et al., 2008). Além disso, a estrutura aromática e o grande número de elétrons $\pi$ delocalizados tornam o Gr promissor para aplicações de descontaminação ambiental, principalmente de sistemas orgânicos (DE MORAES et al., 2019; VENDRAME et al., 2019).

Neste trabalho, avaliamos através de cálculos de primeiros princípios baseados na teoria do Funcional da Densidade, por meio da modelagem computacional de materiais, que consiste em simular ou recriar a realidade física dos átomos de um material a partir de computadores, a $\beta-C D$ e o $\mathrm{Gr}$, como nanomateriais com potencial para absorção ou inclusão de moléculas de ftalatos. Essas simulações levam em consideração a molécula de ftalato BBP interagindo com a $\beta-C D$ ou o $\mathrm{Gr}$, a fim de avaliar as propriedades eletrônicas, energéticas e estruturais dos sistemas resultantes. Os resultados mostram que a interação da $\beta-C D$ ou do $\mathrm{Gr}$ com a molécula de BBP é uma adsorção física (os ftalatos ficam retidos na superfície das nanoestruturas, resultando assim em um "nano-filtro" 
reutilizável). Os valores de energia de ligação são inferiores à 1,00 eV (com BSSE), mostrando que tanto o $\mathrm{Gr}$ como a $\beta-\mathrm{CD}$ podem ser mediadores para a remoção da molécula BBP.

\section{METODOLOGIA}

Para a avaliação das propriedades energéticas, estruturais e eletrônicas da molécula de BBP com o Gr ou $\beta$-CD foi realizada a utilização de cálculos de primeiros princípios com o código computacional SIESTA (SOLER et al., 2002), baseado na Teoria do Funcional da Densidade (DFT). Nestes cálculos, funções de base dupla mais uma função polarizada (DZP) foram usadas. O potencial para correlação de troca foi descrito por LDA (PERDEW; ZUNGER, 1981). A densidade de carga foi representada usando um raio de corte de 200 Ry para a grade de integração no espaço real. Otimizações estruturais foram determinadas usando um gradiente conjugado de modo que as forças residuais fossem menores que 0,05 eVIÅ em todas as coordenadas atômicas. Para realizar simulações de grafeno com a molécula BBP, foram utilizadas condições de contorno periódicas onde a célula tem dimensões de 24,68x 14,85 x 40,00 $\AA^{3}$. O sistema do $\mathrm{Gr}$ consiste em 144 átomos, já a $\beta-C D$ possui 147 átomos. Cálculos semelhantes foram usados em trabalhos recentes de nosso grupo (DE MORAES et al., 2019; FIGUEIREDO et al., 2016; SAINI; FAGAN; TONEL, 2021; TONEL; ZANELLA; FAGAN, 2021; VENDRAME et al., 2019).

Para o cálculo da energia de ligação, usamos a equação (1) (método direto) e a equação (2) considerando o método BSSE (Erro de Superposição do Conjunto de Base) (BOYS; BERNARDI, 1970):

$$
\begin{gathered}
E_{b}=[E(A+B)-E(A)-E(B)] \\
E_{\text {bsse }}=\left[E(A+B)-E\left(A+B_{\text {ghost }}\right)-E\left(A_{\text {ghost }}+B\right)\right]
\end{gathered}
$$


no método de BSSE, a correção é realizada a partir da geometria inicial do sistema $A B$ e calculando a energia total do sistema $A$, considerando todo o conjunto de funções de base, onde o conjunto de funções de base B está localizado na posição correspondente ao sistema $\mathrm{B}$, sem a presença explícita de átomos, e o mesmo ocorre no cálculo do sistema B. Vale ressaltar que valores de energia positiva indicam que o sistema é atrativo.

\section{RESULTADOS E DISCUSSÕES}

Inicialmente, avaliamos as estruturas de $\beta-C D, G r$ e BBP de forma isolada (Figura 1). A $\beta-C D$ possui uma diferença HOMO (orbital molecular mais ocupado)/LUMO (orbital molecular mais baixo desocupado) de 5,72 eV, próximo ao descrito na literatura com metodologia semelhante (FIGUEIREDO et al., 2016). O plot da carga no HOMO e no LUMO ficam sobre os anéis centrais da $\beta-C D$. Já no caso do BBP apresenta diferença HOMO-LUMO de 3,44 eV, e o plot da carga no HOMO e LUMO ficam sobre a região de maior curvatura da molécula. O grafeno puro apresenta um gap nulo e o plot da carga no HOMO e LUMO ficam distribuídos uniformemente sobre a superfície.

Figura 1: Estruturas isoladas: (a) ftalato BBP, (b) $\beta-C D$ e (c) grafeno.

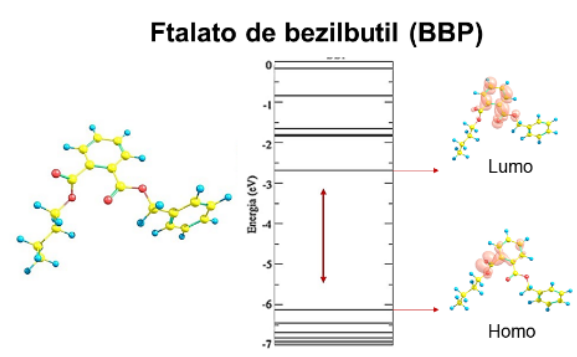

(a)

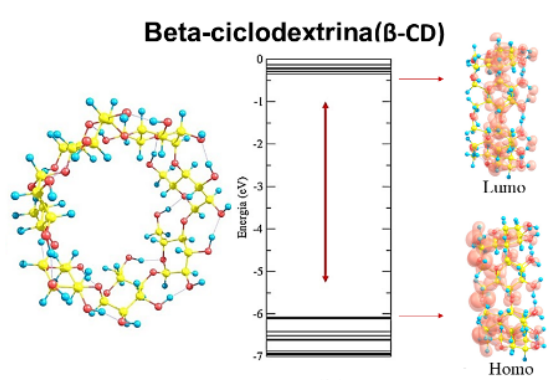

(b)

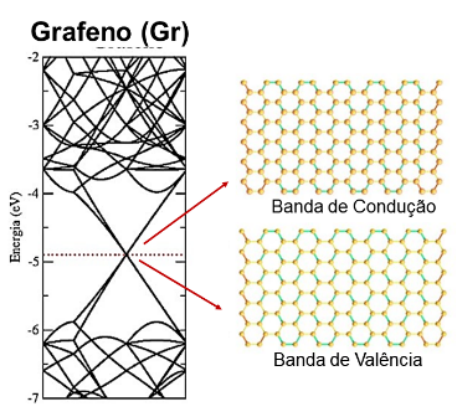

(c) 
Fonte: Construção do autor.

Para a interação da molécula BBP com a $\beta$-CD propomos 6 configurações diferentes. Onde testamos a molécula de ftalato na maior e na menor cavidade do $\beta-C D$, além da parte externa. Já com o $\mathrm{Gr}$ propomos 5 configurações diferentes. $\mathrm{Na}$ Figura 2 apresentamos as configurações para a interação da $\beta-C D$ e do grafeno com a molécula BBP. Já na Tabela 1 encontram-se os valores de energia de interação (eV) (com e sem correção de BSSE), transferência de carga ( $\left.\mathrm{e}^{-}\right)$, menor distância entre as estruturas $(\AA)$ e a diferença HOMO/LUMO (eV). Finalmente, os níveis energéticos encontram-se na Figura (3-a).

Figura 2: Configurações mais estáveis estudadas para a interação da molécula de ftalato (BBP) com: (a) $\beta-C D$ e (b) grafeno.

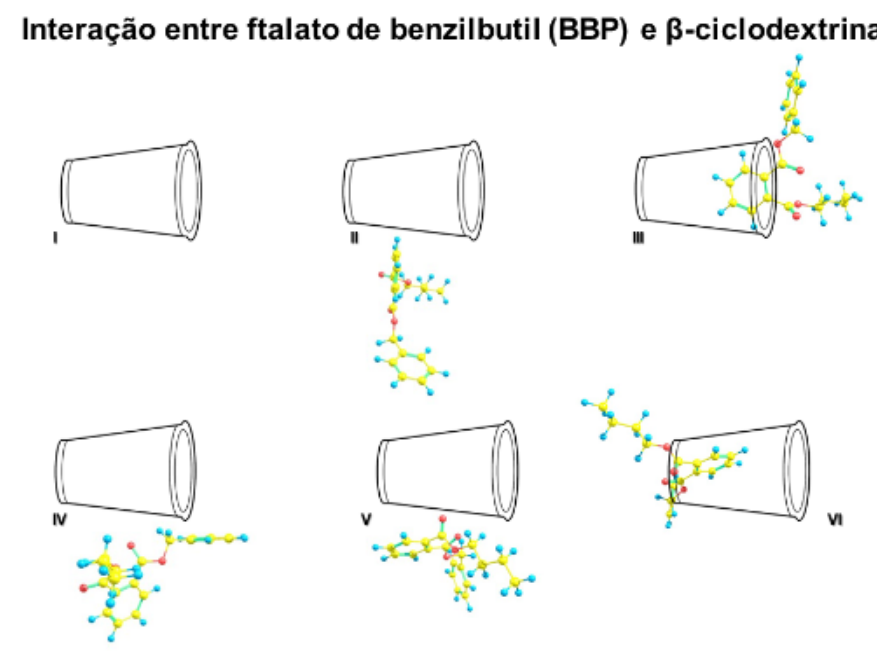

Interação entre ftalato de benzilbutil (BBP) e grafeno (Gr)

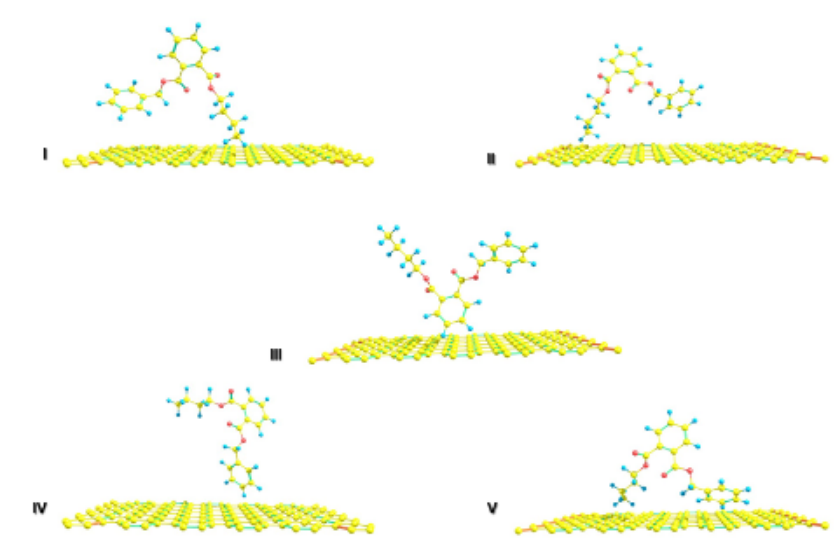


Fonte: Construção do autor.

Tabela 1: Configurações estudadas para a interação da molécula de ftalato (BBP) com o grafeno ou beta-ciclodextrina. Energia de ligação sem e com correção da base por BSSE, menor distância de ligação, diferença HOMO/LUMO e transferência de carga.

\begin{tabular}{|c|c|c|c|c|c|c|}
\hline Estrutura & Configuração & $\begin{array}{c}\mathrm{E}_{\mathrm{b}}(\mathrm{eV}) \\
(\text { método } \\
\text { direto })\end{array}$ & $\begin{array}{c}\mathrm{E}_{\mathrm{bsse}}(\mathrm{eV}) \\
(\mathrm{BSOm}) \\
\mathrm{BSSE})\end{array}$ & $\begin{array}{c}\text { Menor } \\
\text { distância } \\
(\mathrm{Ang})\end{array}$ & $\begin{array}{c}\Delta \text { Homo/ } \\
\text { Lumo } \\
(\mathrm{eV})\end{array}$ & $\begin{array}{c}\text { Transferência } \\
\text { de carga (e) }\end{array}$ \\
\hline$\beta-\mathrm{CD}$ & - & - & - & - & 5,72 & - \\
\hline $\mathrm{BBP}$ & - & - & - & - & 3,44 & - \\
\hline$\beta-\mathrm{CD}+\mathrm{BBP}$ & $\mathrm{I}$ & -0.14 & -0.09 & 1.75 & -3.32 & -0.01 \\
\hline$\beta-\mathrm{CD}+\mathrm{BBP}$ & $\mathrm{II}$ & -0.14 & -0.08 & 2.03 & -3.29 & 0.01 \\
\hline$\beta-\mathrm{CD}+\mathrm{BBP}$ & $\mathrm{III}$ & -1.26 & -0.94 & 1.80 & -2.86 & 0.22 \\
\hline$\beta-\mathrm{CD}+\mathrm{BBP}$ & $\mathrm{IV}$ & -0.75 & -0.54 & 2.21 & -2.91 & 0.01 \\
\hline$\beta-\mathrm{CD}+\mathrm{BBP}$ & $\mathrm{V}$ & -0.65 & -0.39 & 2.16 & -2.64 & 0.13 \\
\hline$\beta-\mathrm{CD}+\mathrm{BBP}$ & $\mathrm{VI}$ & -1.32 & -0.82 & 1.88 & -3.35 & -0.04 \\
\hline Grafeno+BBP & I & -0.23 & -0.15 & 2.94 & -0.86 & 0.05 \\
\hline Grafeno+BBP & II & -0.21 & -0.14 & 3.00 & -0.86 & 0.05 \\
\hline Grafeno+BBP & III & -0.26 & -0.18 & 2.75 & -0.86 & 0.07 \\
\hline Grafeno+BBP & IV & -0.22 & -0.15 & 2.50 & -0.86 & 0.06 \\
\hline Grafeno+BBP & V & -0.70 & -0.42 & 3.00 & -0.86 & 0.09 \\
\hline
\end{tabular}

A interação mais estável da BBP com a $\beta-C D$ ocorre na configuração III, onde a molécula de BBP interage com a parte interna da cavidade maior da $\beta-C D$. Nesse caso obtivemos uma energia de ligação na ordem de 0,94 eV (através do método de correção de base - BSSE) ou de 1,26 eV (método direto). Observamos que ocorre uma diminuição da diferença HOMO-LUMO em relação a molécula isolada 2,85 eV, e a transferência de carga $+0,22$ e-, ou seja, a molécula de BBP recebe carga da $\beta$-CD. Li e colaboradores (2019) demonstram por meio de um trabalho experimental 
e teórico que a molécula de $\beta-C D$ tem tamanho suficiente para a captura com alta seletividade da molécula de BBP.

Já a interação mais estável da molécula de BBP com o grafeno é a configuração $V$, que apresenta energia de ligação com correção de base na ordem de $0,42 \mathrm{eV}$. Nesse sistema a distância de ligação é em torno de $3 \AA$, onde o grafeno doa $+0,09 \mathrm{e}^{-}$de carga para a molécula de ftalato. A interação ocorre num regime de adsorção física, ou seja, os constituintes interagem fracamente.

Santana-Mayor e colaboradores (2018) demonstraram experimentalmente que o grafeno/óxido de grafeno é eficiente para a remoção de quatorze moléculas de ftalatos, entre elas a BBP. Segundo o autor, o nanofiltro baseado no grafeno é uma alternativa inovadora aos métodos convencionais para a remoção das moléculas de ftalatos, e com pequenas quantidades de sorvente é possível atingir boa eficiência de extração, o que está de acordo com os princípios da Química Verde (SANTANA-MAYOR et al., 2018).

Na Figura 3-a apresentamos os níveis energéticos e os plots da cargas no HOMO e LUMO da interação da $\beta$-CD e grafeno com a molécula de ftalato (BBP). Os resultados mostram que na interação da $\beta-C D$ com o BBP não ocorre alterações significativas, apenas uma sobreposição dos níveis indicando que ocorre uma adsorção física. Já que o plot da carga no HOMO fica somente sobre a $\beta-C D$ e no LUMO somente sobre a BBP. No caso da interação do Gr com a molécula de ftalato (Figura 3-b), observamos que não ocorre alteração em relação ao grafeno isolado, indicando uma interação fraca, e o plot da carga tanto no HOMO como no LUMO ficam somente sobre o grafeno. Temos assim, em ambos os casos, uma adsorção física, que é umas das condições necessárias para a utilização do grafeno ou das $\beta-C D$ como nanofiltro da molécula de ftalato BBP. 
Figura 3: (a) Níveis energéticos da interação da $\beta-C D$ e (b) e bandas energéticas $G r$ interagindo com a molécula de ftalato (BBP).
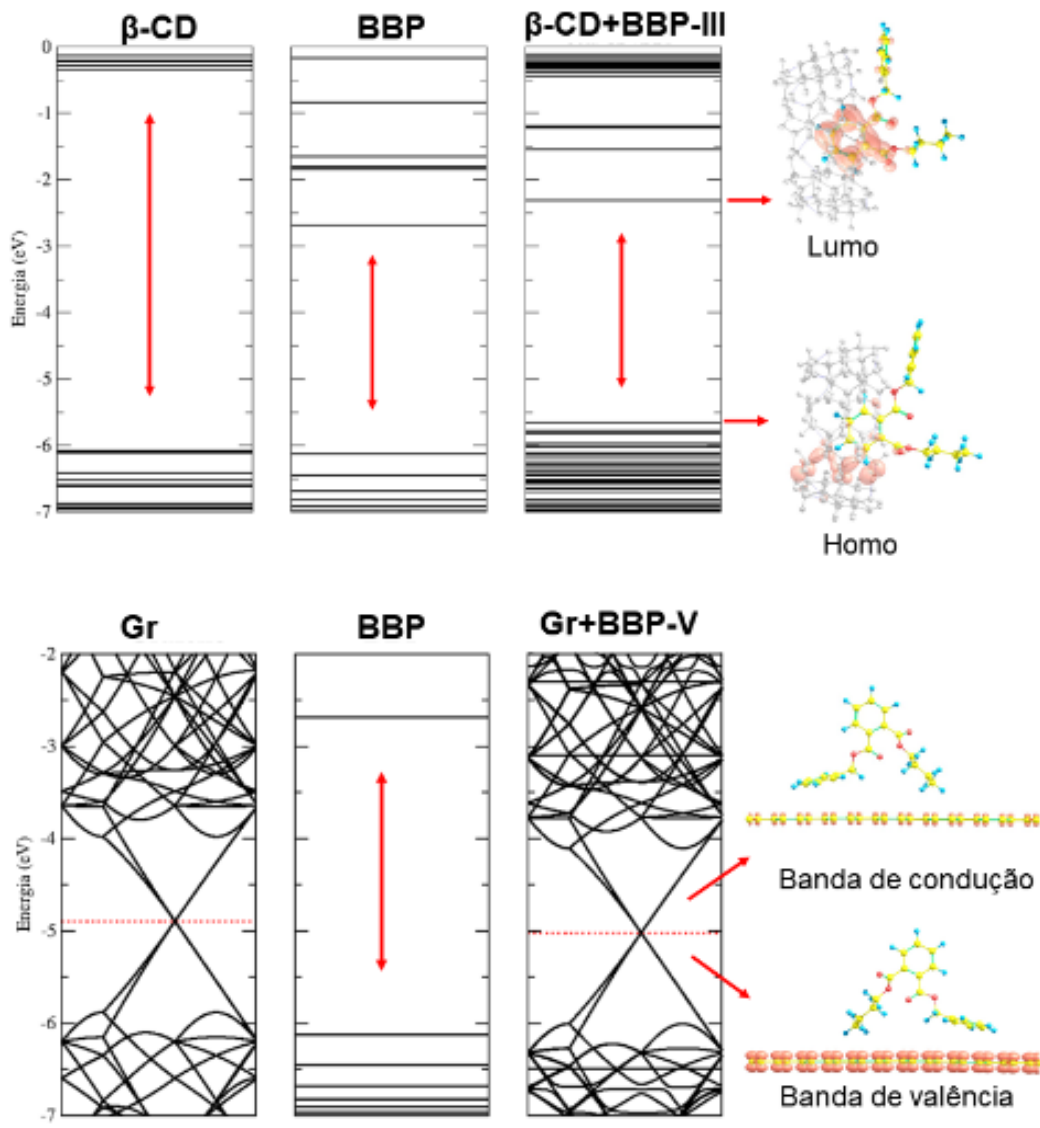

Fonte: Construção do autor

\section{CONCLUSÃO}

Realizamos cálculos usando cálculos de primeiros princípios com base no DFT para avaliar a interação da molécula de ftalato BBP com o $\mathrm{Gr}$ e com a $\beta$-CD. Os resultados das propriedades energéticas, estruturais e eletrônicas estimam que as interações ocorrem via regime de adsorção física, ou seja, a interação que ocorre é fraca sem modificações nas propriedades eletrônicas e estruturais dos agentes interagentes. No caso do Gr interagindo com a molécula de BBP obtivemos como energia mais estável $0,42 \mathrm{eV}$. Já no caso da $\beta-C D$ interagindo com BBP a energia mais estável, dentre as 6 configurações, foi de $0,94 \mathrm{eV}$. No entanto, todas as energias de ligação foram inferiores a $1,00 \mathrm{eV}$, indicando um regime de adsorção 
física. Vale ressaltar que em todas as configurações estudadas os valores de energia de ligação foram mais baixos com o método de correção de BSSE, indicando que no método direto existe uma sobreposição das bases o que acarreta num maior valor de energia de ligação. A ausência de ligações químicas entre os sistemas é uma das condições necessárias para a utilização de grafeno e da beta-ciclodextrina como possíveis plataformas de nanofiltros da molécula de BBP.

\section{AGRADECIMENTOS}

Os autores agradecem ao CENAPAD-SP (Centro Nacional de Processamento de Alto Desempenho em São Paulo) e à UFN (Universidade Franciscana) pelo espaço computacional, e às agências brasileiras CNPQ, CAPES e Fapergs pelo apoio financeiro.

\section{REFERÊNCIAS}

BENJAMIN, S. et al. Phthalates impact human health: Epidemiological evidences and plausible mechanism of action. Journal of Hazardous Materials, v. 340, p. 360-383, out. 2017.

BOYS, S. F.; BERNARDI, F. The calculation of small molecular interactions by the differences of separate total energies. Some procedures with reduced errors.

Molecular Physics, v. 19, n. 4, p. 553-566, out. 1970.

CHEN, L. et al. Exposure assessment of phthalates in non-occupational populations in China. Science of The Total Environment, v. 427-428, p. 60-69, jun. 2012.

DE MORAES, E. E. et al. Density functional theory study of m-aromatic interaction of benzene, phenol, catechol, dopamine isolated dimers and adsorbed on graphene surface. Journal of Molecular Modeling, v. 25, n. 10, p. 302, out. 2019.

DEL VALLE, E. M. M. Cyclodextrins and their uses: a review. Process

Biochemistry, v. 39, n. 9, p. 1033-1046, maio 2004.

DUAN, X.-L. et al. Benzyl butyl phthalate (BBP) triggers the malignancy of acute myeloid leukemia cells via upregulation of PDK4. Toxicology in Vitro, v. 62, p. 104693, fev. 2020.

FIGUEIREDO, J. et al. Interaction of $\langle|>\alpha</|>-$ Tocopherol with $<|>\alpha</|>$ - and $<|>\beta</|>-C y c l o d e x t r i n s:$ A First-Principles Investigation. Journal of

Nanopharmaceutics and Drug Delivery, v. 3, n. 1, p. 70-76, 1 mar. 2016. GONZÁLEZ-SÁLAMO, J.; SOCAS-RODRÍGUEZ, B.; HERNÁNDEZ-BORGES, J. Analytical methods for the determination of phthalates in food. Current Opinion in Food Science, v. 22, p. 122-136, ago. 2018.

HEDGES, A. R. Industrial Applications of Cyclodextrins. Chemical Reviews, v. 98, n. 5, p. 2035-2044, jul. 1998. 
HU, J.; YANG, Q.; WANG, J. L. Biodegradation of di-n-butyl phthalate in sequencing batch reactor bioaugmented with Micrococcus sp. and the bacterial community analysis. International Journal of Environmental Science and Technology, v. 12, n. 9, p. 2819-2828, set. 2015.

KAEWLAOYOONG, A. et al. Occurrence of phthalate esters around the major plastic industrial area in southern Taiwan. Environmental Earth Sciences, v. 77, n. 12, p. 457, jun. 2018.

LI, J. et al. $\beta$-Cyclodextrin-Stabilized Au Nanoparticles for the Detection of Butyl Benzyl Phthalate. ACS Applied Nano Materials, v. 2, n. 5, p. 2743-2751, 24 maio 2019.

NOVOSELOV, K. S. Electric Field Effect in Atomically Thin Carbon Films. Science, v. 306, n. 5696, p. 666-669, 22 out. 2004.

OKAMOTO, Y.; UEDA, K.; KOJIMA, N. Potential Risks of Phthalate Esters:

Acquisition of Endocrine-disrupting Activity during Environmental and Metabolic Processing. Journal of Health Science, v. 57, n. 6, p. 497-503, 2011.

PERDEW, J. P.; ZUNGER, A. Self-interaction correction to density-functional approximations for many-electron systems. Physical Review B, v. 23, n. 10, p. 5048-5079, 15 maio 1981.

RADKE, E. G. et al. Phthalate exposure and metabolic effects: a systematic review of the human epidemiological evidence. Environment International, v. 132, p. 104768, nov. 2019.

SAINI, S. S.; FAGAN, S. B.; TONEL, M. Z. A novel and green extraction strategy for sensitive determination of phthalates in aqueous samples: Analytical and computational studies. Microchemical Journal, v. 166, p. 106227, jul. 2021. SANTANA-MAYOR, Á. et al. Reduced graphene oxide-coated magnetic-nanoparticles as sorbent for the determination of phthalates in environmental samples by micro-dispersive solid-phase extraction followed by ultra-high-performance liquid chromatography tandem mass spectrometry. Journal of Chromatography A, v. 1565, p. 36-47, ago. 2018.

SOLER, J. M. et al. The SIESTA method for $a b$ initio order- $N$ materials simulation. Journal of Physics: Condensed Matter, v. 14, n. 11, p. 2745-2779, 25 mar. 2002. TERRONES, M. et al. Graphene and graphite nanoribbons: Morphology, properties, synthesis, defects and applications. Nano Today, v. 5, p. 351-372, 2010.

TONEL, M. Z.; ZANELLA, I.; FAGAN, S. B. Theoretical study of small aromatic molecules adsorbed in pristine and functionalised graphene. Journal of Molecular Modeling, v. 27, n. 6, p. 193, jun. 2021.

VENDRAME, L. F. O. et al. Nanofilter based on functionalized carbon nanostructures for the adsorption of pentachlorophenol molecules. Computational and Theoretical Chemistry, v. 1165, p. 112561, out. 2019.

ZANELLA, I. et al. Chemical doping-induced gap opening and spin polarization in graphene. Physical Review B, v. 77, n. 7, 20 fev. 2008.

ZHENG, X.; ZHANG, B.-T.; TENG, Y. Distribution of phthalate acid esters in lakes of Beijing and its relationship with anthropogenic activities. Science of The Total Environment, v. 476-477, p. 107-113, abr. 2014. 
EDUCAÇÃO, SAÚDE

ETECNOLOGIA

26 A 28 DE OUTUBRO DE 2021

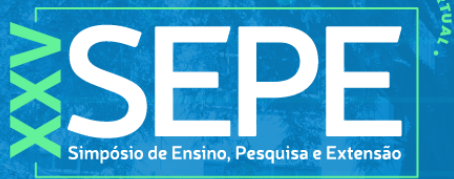

QUFN

ZHOU, J.; CAI, Z.-H.; XING, K.-Z. Potential mechanisms of phthalate ester embryotoxicity in the abalone Haliotis diversicolor supertexta. Environmental Pollution, v. 159, n. 5, p. 1114-1122, maio 2011. 